\title{
Téoros
}

Revue de recherche en tourisme

\section{Les pourvoiries du Québec}

\section{Robert Soubrier}

Volume 8, numéro 2, juillet 1989

\section{Hébergement et tourisme}

URI : https://id.erudit.org/iderudit/1080324ar

DOI : https://doi.org/10.7202/1080324ar

Aller au sommaire du numéro

\section{Éditeur(s)}

Université du Québec à Montréal

\section{ISSN}

0712-8657 (imprimé)

1923-2705 (numérique)

Découvrir la revue

Citer cet article

Soubrier, R. (1989). Les pourvoiries du Québec. Téoros, 8(2), 26-29.

https://doi.org/10.7202/1080324ar d'utilisation que vous pouvez consulter en ligne.

https://apropos.erudit.org/fr/usagers/politique-dutilisation/ 


\section{Les pourvoiries du Québec $^{(1)}$}

L'attrait des Québecois el des touristes nonrésidents pour les activites de chasse et de peche sur le territoire du Québec a conduit en 1985 le ministère du Loisir, de la Chasse et de la Pêche et le ministère du Tourisme à s'interroger sur la capacité des infrastructures mises à la disposition des chasseurs, des pêcheurset des trappeurs à rêpondre à leurs besoins. Aussi, afin de favoriser la creation d'une industrie touristique en arrière-pays fondée sur l'exploitation des ressources fauniques capable de retenir les touristes québécois et d'attirer davantage une clientèle internationale, ces deux ministères ont confié le mandat au Departement des sciences du loisir de IUQTR de réaliser une étude pour:

1- dégager la personnalité touristique de chaque pourvoirie;

2- realiser un systeme de classification de l'hébergement, de la restauration el des activités de chasse et de pêche;

3- mettre à jour le répertoire des pourvoiries du Quebec.

Cet article vise done 1) a définir le nombre de pourvoiries par régions, 2) à présenter très sommairement la methode qui a eté proposée pour étublir le système de catégorisation et de classification de l'hébergement et de la restauration et 3) à situer les pourvoiries du Québec par rapport aux méthodes de catégorisation et de classification qui ont été proposées.

\section{Les pourvoiries et le territoire québécois}

Les pourvoiries du Québec sont éparpilllées dans les 10 régions(2) administratives à trawers les $1648000 \mathrm{~km} 2$ de superficie du territoire québécois. Au moment de l'enquête, on dénombrait 499 pourvoiries et 3815 unités (camps, chalets, dortoirs, etc.). Le tableau 1 présente le nombre de pourvoiries visitées selon la région administrative. On remarque une concentration plus importante de pourvoiries dans les régions de IOutaouais, I'Abitibi/Témiscamingue et la Côte-Nord. A elles seules, ces trois régions regroupent $60,3 \%$ des pourvoiries du Quebec.

\section{La méthodologie}

* Mansieur Robert Soubrier est specialise dans le domaine de la planification et de l'amenagement des equipements de loisirs. II est présentement Directeur du Departement des Sciences du loisir de FUniversité du Québec à Trois-Riviéres.
Le mandat confiè à l'équipe de recherche consistait a proposer unsystène de classification fondé sur des normes prétablies conjointement par le M.L.C.P., le M.T. et I'Association des pour- voyeurs du Québec. À la suggestion de l'équipe de recherehe, les differents intervenants dans le dossier ont accepté de distinguer la catégorisation de la classification, la premiere permettant détablir des types, niveaux ou groupes homogènes, mutuellement exclusifs et la seconde, d'élaborer un système de pondération pour déterminer des seuils hiếrarchiques dequal ité. En tenant compte de ces distinctions, les méthodes de catégorisationet de classification élaborées sont identiques pour l'hébergement et la restauration. Seuls les paramètres changent pour s'adapterà la spécificité des dimensions ou volets ă évaluer.

L'équipe de recherche a privilégié un mode de calcul qui permet d'obtenir une cote globale pour chaque ensemble d'unités d'hébergement ou de restauration (appelé descripteur) ainsi qu'une cole par unité d'hébergement ou de restauration. Deux scénarios presque opposés ont êté proposés pour permettre aux décideurs de faire des choix plus eclairés. Le premier scénario se fonde sur le ealcul des moyennes et le second sur celui de la cote la plus basse.

\section{La catégorisation}

La catégorisation correspond aux différents niveaux de services ou d'équipements relatifs à l'hébergement ou å la restauration mis à la disposition des usagers dans chaque pourvoirie du réseau québecois. Concrêtement, cela signifie que durant la phase dimventaire de chaque pourvoirie, les évaluateurs ont recensé systênatiquement tous les services ou équipements mis à la disposition du villegiateur. Les tableaux 2 et 3 presentent les composantes du modèle opératoire utilisé. Le réseau d'hébergement est constitué des camps, des chalets, des auberges forestières, des motels et des dortoirs. Celui de la restauration, des auberges forestières, des pavillons et des restaurants.

Chaque unité du réseau â ếe catégorisće de façon indépendante selon la quantité de services. et d'equipements mis à la disposition du villêgiateur, Pour catégoriser l'ensemble des unités d'un même wolet (par exemple les chalets), deux scénarios ont été présentés. Dans le premier, on calcule la moyenne des cotes obtenues pour chaque unité d'un même volet pour déterminer la categorie à laquelle appartient cet ensemble. Dans le second, la cote la plus basse obtenue pour une unité détermine la catégorie à laquelle appartient cet ensemble. 
TABLEAU 1

Nombre de pourvoiries visitées selon la région administrative Été 1985

\begin{tabular}{|l|c|c|}
\hline \multicolumn{1}{|c|}{ Régions } & Nombre & $\%$ \\
\hline 1- Bas St-Laurent & 9 & 1.8 \\
2- Saguenay/Lac St-Jean & 32 & 6,4 \\
3- Québec & 37 & 7,4 \\
4- Trois-Rivières & 49 & 9,8 \\
5- Estrie & 7 & 1,4 \\
6- Montréal & 30 & 6,0 \\
7- Outaouais & 134 & 26,9 \\
8- Abitibi/Témiscamingue & 97 & 19,4 \\
9- Côte-Nord & 70 & 14,0 \\
10- Baie James/Nord québecois & 35 & 6,8 \\
TOTAL: & 499 & \\
\hline
\end{tabular}

\section{TABLEAU 2}

Volet "hébergement" Composantes du modèle opératoire pour la catégorisation

\begin{tabular}{|c|c|c|c|c|}
\hline Descripteurs & Éléments & Indicateurs & Mesures: & Modalités* \\
\hline $\begin{array}{l}\text { Camps } \\
\text { Chalets } \\
\text { Auberges forestieres }\end{array}$ & $\begin{array}{l}\text { Services } \\
\text { Espace } \\
\text { disponible par } \\
\text { usager }\end{array}$ & $\begin{array}{l}\text { Eau courante froide } \\
\text { Eau courante chaude } \\
\text { Toilette(s) extéricure(s) } \\
\text { Toilette(s) intérieure(s) } \\
\text {. Fosse septique } \\
\text { Prise(s) d'électricité } \\
\text {. Foyer intérieur } \\
\text {. Douche(s) avec eau chaudd } \\
\text {. Laveuse(s) accessible(s) } \\
\text { à la clientèle } \\
\text { Sécheuse(s) accessible } \\
\text { a la clientéle } \\
\text { Cuisine ou cuisinette } \\
\text { Réfrigérateur } \\
\text { Congélateur } \\
\text { Service de glace } \\
\text { Service de premiers soins } \\
\text { Literie (foumie à } \\
\text { l'hébergement) } \\
\text { Superficie des unités } \\
\text { dhébergement } \\
\text { Nombre de couches }\end{array}$ & $\begin{array}{l}\text { N. de prises } \\
N \text { de robinets } \\
N \\
N \\
N \\
N \text {, de prises } \\
N \\
N \\
N \\
N \\
N \\
N \\
\text { P/A } \\
\text { P/A }\end{array}$ & $\begin{array}{l}\text { Chiffre absolu } \\
\text { Chiffre absolu }\end{array}$ \\
\hline
\end{tabular}

* P/A = présence ou absence: $\mathrm{N}=$ nombre

\section{La classification}

La classification reflète le niveau de qualité ou l'état de l'hébergement ou de la restauration offert par le réseau des pourvoiries du Québec. Elle découle de l'application adaptée d'un document réalisé par un groupe de travail formé des représentants du MLCP, du MT et de l'Association des pourvoyeurs du Québec dans lequel étaient précisées les normes de classification des pourvoiries, En s'appuyant sur ces normes. l'équipe de recherche a constitué une échelle d'évaluation en quatre points afin de pouvoir différencier les pourvoiries de bas ou de haut de gamme.
Toutes les unités d'hébergement ou de restauration ont été classifiées en calculant la moyenne des cotes obtenues pour chaque élément évalué dans chaque unité. La classification de l'ensemble des unités d'un même type dans une mệme pourvoirie a été rếaliśce selon deux scénarios. Dans le premier scénario, on a calculé la moyenne des cotes obtenues pour les unités d'un même volet. Dans le second, les calculs ont été réalises tés la cote la plus basse obtenue dans cet ensemble d'unités. Les tableaux 4 et 5 présentent les composantes des descripteurs des volet hébergement et restauration qui ont servi ă la classification. en accordant à chaque volet ou ensemble d'uni-

\section{Situation des pourvoiries par rapport aux catégories et clas- ses}

Les chalets et les camps constituent à eux seuls $96,8 \%$ de toutes les unités d'hébergement des pourwoiries du Québec. Toutefois, même si les auberges forestières ne représentent que $2,1 \%$ des unités d'hébergement, elles n'en constituent pas moins la ressource principale du développement d'une industrie touristique en arrière pays. On remarque en effet dans le tableau 6 qu'audelà de $53,3 \%$ des auberges forestières offrent une variété de services supérieurs (catégories III et IV selon la cote la plus basse) alors que seulement $12,2 \%$ des camps et $23,8 \%$ des chalets atteignent un tel niveau de service. Le mode d'hébergement dans les motels et les dortoirs paraît négligeable tant sur le plan de la quantité des unités d'hébergement $(1,1 \%)$ que sur celui des services offerts à̀ la clientèle. Il faut également noter que le bateau représente un mode d'hébergement rarissime qui n'avait d'ailleurs pas été prévu comme tel lors de l'élaboration des instruments d'inventaire. Ce type d'hébergement pourrait, tout comme les auberges forestières, devenir un équipement recherché si l'on se fie à loriginalité de lexpérience récréative qu'il permet sur certains plans d'eau. Ceci demeure néanmoins à vérifier d'une façon plus particulière.

La restauration présente des caractéristiques différentes de l'hébergement puisque à l'inverse de celui-ci, ill n'existe généralement dans une pourvoirie qu'un seul lieu où il est possible de se restaurer. Conséquemment, il ne peut y avoir de catégorisation par la cote la plus basse. La moyenne des résultats fournis dans le tableau 7 indique done la proportion de pourvoiries qui offrent les services indiqués. Seulement $35,7 \%$ des établissements offrent un tel service et de ce pourcentage, seulement $4,4 \%$ des pourvoiries permettent de prendre une consommation et un repas avec un service complet aux tables. En tait, les auberges forestieres constituent $32 \%$, les pavillons $63,1 \%$, les restaurants $4,9 \%$ des établissements où un service de restauration est offert à la clientèle. Cela démontre toute l'importance de ces équipements pour établir une industric touristique attirante autant pour les visiteurs étrangers que pour la population vieillissante et économiquement avantagée des chasseurs et pêcheurs québécois et canadiens pour qui le confort et la sécurité peuvent devenir des facteurs conditionnant la poursuite de ce genre dauctivité.

L'intérât de doubler un système de catégorisation par une classification reflétant non pas les niveaux de services disponibles dans une pourvoirie mais la qualité de ce qui est mis à la disposition d'un villégiateur lui assure un contrôle minimal sur le confort qu'il desire obtenir par rapport à sa capacité de payer et à l'expé= rience récréative à laquelle il aspire. 
Ainsi, pour l'hébergement, le tableau 8 montre que $78,4 \%$ de tous les camps et $60 \%$ de tous les chalets visités se situeraient dans les classes I et II si on utilise la moyenne, et respectivement $95,6 \%$ pour les camps et $78,6 \%$ pour les chalets si on calcule avec la cote la plus basse. Par contre, $72,5 \%$ (calculé avec la moyenne) et $67,7 \%$ (calcule avec la cote la plus basse) des auberges forestières se situent dans les catégories III et IV.

Le tableau 9 montre que și l'on avait appliqué le système de classification pour la restauration $80,8 \%$, calculé avec la moyenne, ou $64,4 \%$, calculé avec la cote la plus basse, des auberges forestières se seruient situées dans la classe III et IV. Cette proportion reste également très élevée pour les pavillons et les restaurants.

\section{Conclusion}

Lastructuration du système d'hébergement et de restauration des pourvoiries du Québec montre que le villégiateur à accès à une très grande gamme de services et de niveaux de qualité. Dans un contexte où l'individu recherche un plus grand contact avee la nature el une experience dans laquelle le prélèvement des ressources fauniques constitue le motif principal de deplacement, il doit pouvoir choisir lé lieu en relation avec l'expérience qu'il recherche. Depuis 1987. le Répertoire des pourvoiries du Québec contient une série d'informations relatives aux expériences récréatives et aux services ofterts dans chaque pourvoirie, de même qu'une catégorisution des unités dhébergement. Pour le bénéfice des usagers potentiels et le développement d'une véritable industrie en arrière-pays, il serait souhaitable que le MLCP, le MT et l'Association des pourvoyeurs du Québec complètent le systè̀me de catểgorisation et de classification afin d'inciter le pourvoyeur à offrir des services de qualité superieure.

\section{NOTE EXPLICATIVE}

(1) Ce teste es un résumé tres succinct d'un rapport intitules Inventuire et classiricution des pourvoiries de chasse et de peche du Quibec (1986), fune étude realisce poner le MLCP at le MT. Elle fut dirigete par Gilles

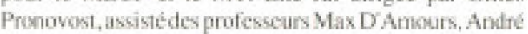
Burahe at Rebert Soubrier. Liateur de cer article avait la responsibilité d'elliborer les syotentes de classifica tion et de catégotisalion pour bes wolets hehergement at resauration des pourvoiries du Quekes.
TABLEAU 3

Volet "restauration" Composante du modèle opératoire pour la catégorisation

\begin{tabular}{|c|c|c|c|c|}
\hline Deseripteurs & Eléments & Indicateurs & Mesures: & Modalités" \\
\hline $\begin{array}{l}\text { Auberges } \\
\text { forestieres } \\
\text { Pavillons } \\
\text { Restaurants }\end{array}$ & 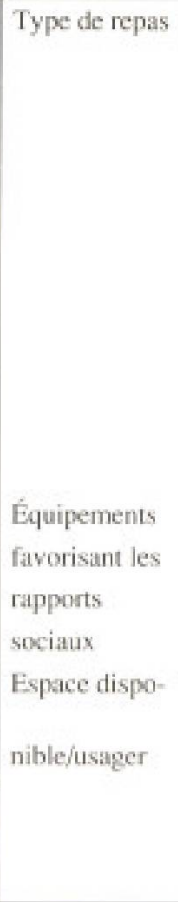 & 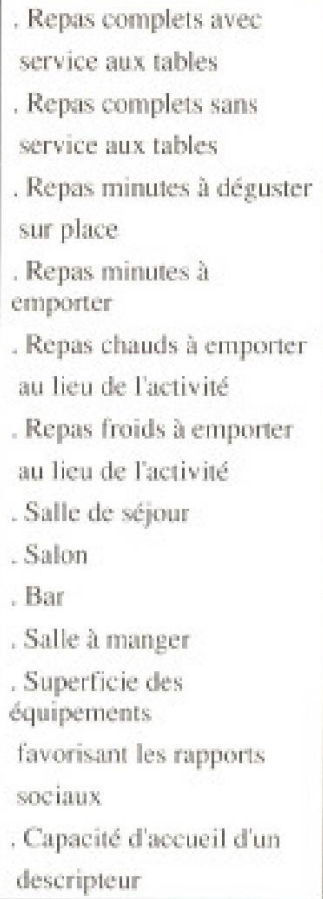 & $\begin{array}{l}\text { P/A } \\
\text { P/A } \\
\text { P/A } \\
\text { P/A } \\
\text { P/A } \\
\text { P/A } \\
\text { P/A } \\
\text { P/A } \\
\text { P/A } \\
\text { Metres carres } \\
\text { de l'espace } \\
\text { Nombe de } \\
\text { Persones }\end{array}$ & Chiffre absolu \\
\hline
\end{tabular}

* $\mathrm{P} / \mathrm{A}=$ pésenciabsence; $\mathrm{N}=$ nomber

\section{TABLEAU 4}

Volet "hébergement" Composantes des descripteurs pour la classification

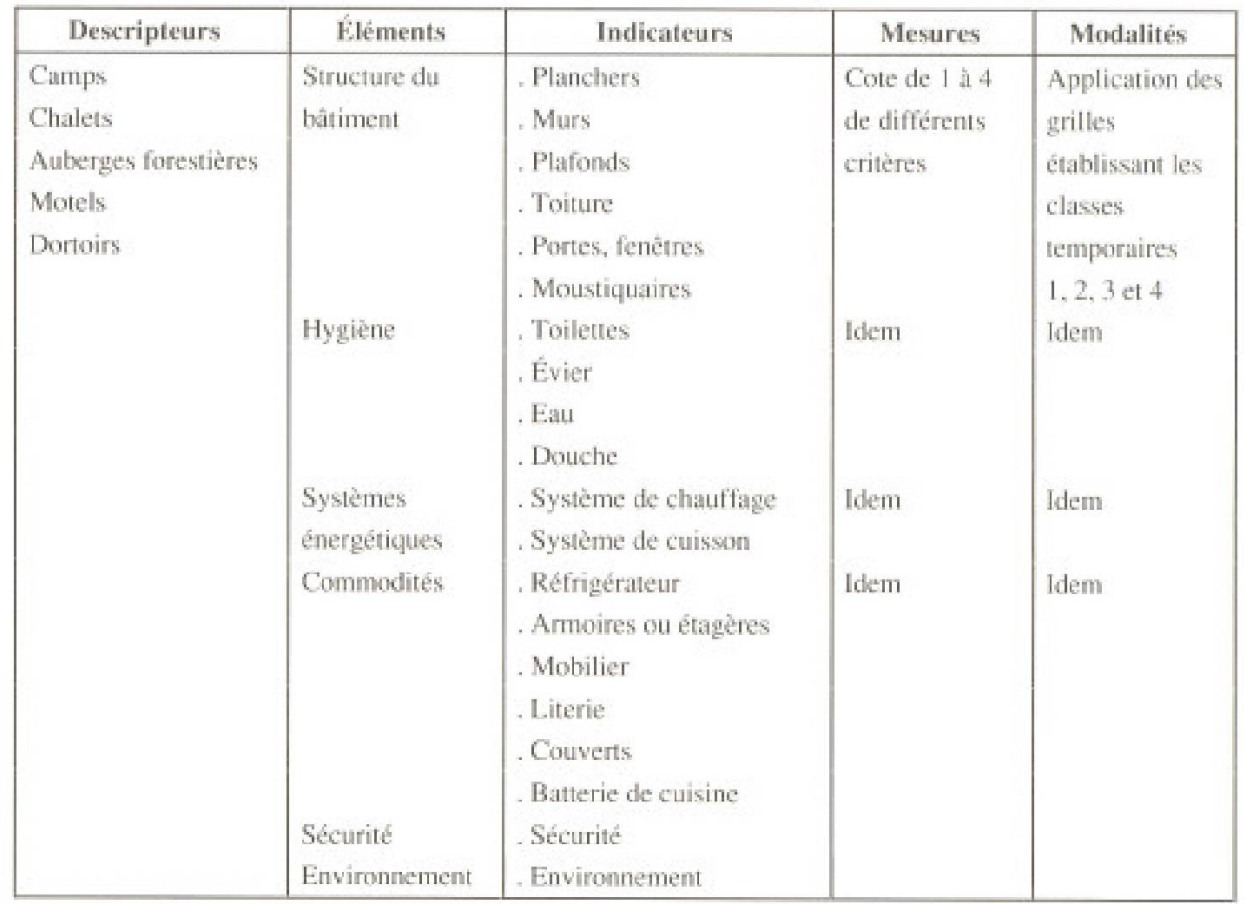


TABLEAU 5

Volet "restauration" Composantes: modèle opératoire pour la classification

\begin{tabular}{|c|c|c|c|c|}
\hline Destripterss & EHements & Indicateurs & Mesures & Muralites \\
\hline 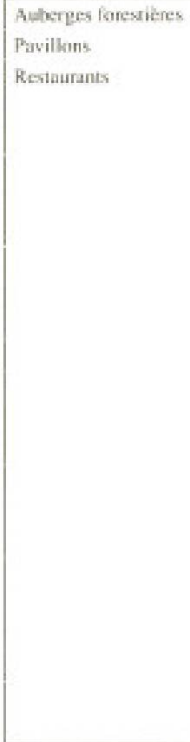 & $\begin{array}{l}\text { Salkin } \\
\text { Har } \\
\text { Salle in mizngur } \\
\text { Cuisine }\end{array}$ & 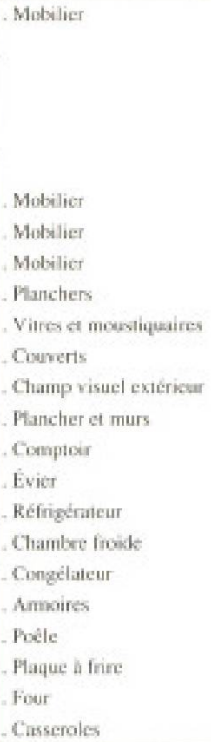 & 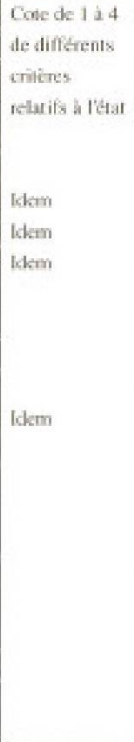 & 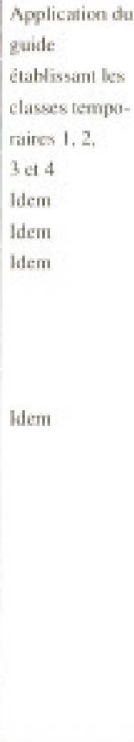 \\
\hline
\end{tabular}

\section{TABLEAU 6}

Résultats comparés pour l'ensemble des pourvoiries du Québec incluses dans chaque catégorie selon la méthode de la moyenne et celle de la cote la plus basse

\begin{tabular}{|c|c|c|c|c|c|c|c|c|c|c|}
\hline \multirow[t]{2}{*}{ Calcharies } & \multicolumn{2}{|c|}{$\begin{array}{l}\text { Cimps } \\
\mathrm{N}=\|\| 05\end{array}$} & \multicolumn{2}{|c|}{$\begin{array}{l}\text { Chalets } \\
N=2418\end{array}$} & \multicolumn{2}{|c|}{$\begin{array}{l}\text { Auberges } \\
\text { forestitires } \\
\mathrm{N}=72\end{array}$} & \multicolumn{2}{|c|}{$\begin{array}{l}\text { Modrls } \\
N=9\end{array}$} & \multicolumn{2}{|c|}{$\begin{array}{l}\text { Dortuirs } \\
\mathrm{N}=\text { गI }\end{array}$} \\
\hline & t. & $\begin{array}{l}+ \\
\text { B } 8\end{array}$ & & is & & " & $\begin{array}{l}4 \\
\times 40 \\
\end{array}$ & $\begin{array}{l}4 \\
\mathrm{~B}\end{array}$ & $\times$ & $\begin{array}{l}\text { 中 } \\
\text { B } \%\end{array}$ \\
\hline 1 & 32,2 & 50,6 & 28,0 & 478 & 16,4 & 160 & 37.5 & 375 & 33.3 & 3.3 \\
\hline III & 52.9 & 37,2 & 40.3 & 28.4 & 28,0 & 30.7 & 12.5 & 250 & 333 & 26.7 \\
\hline III & 13,4 & 10,7 & 25,5 & 18,2 & 14,7 & 120 & 25,01 & 12.5 & 333 & 93.2 \\
\hline IV & 1.5 & 1.5 & 8,2 & 5.6 & 41,3 & 41.3 & 25.9 & 280 & b. & 6.7 \\
\hline
\end{tabular}

* $\mathrm{x}=$ calcule ave la methoude de la moyene

$\mathrm{B}=$ calcule avec la methode de ti cotie la plua basse
TABLEAU 7

Fréquences et pourcentages de l'ensemble des pourvoiries du Québec incluses dans chaque catégorie par la méthode des moyennes

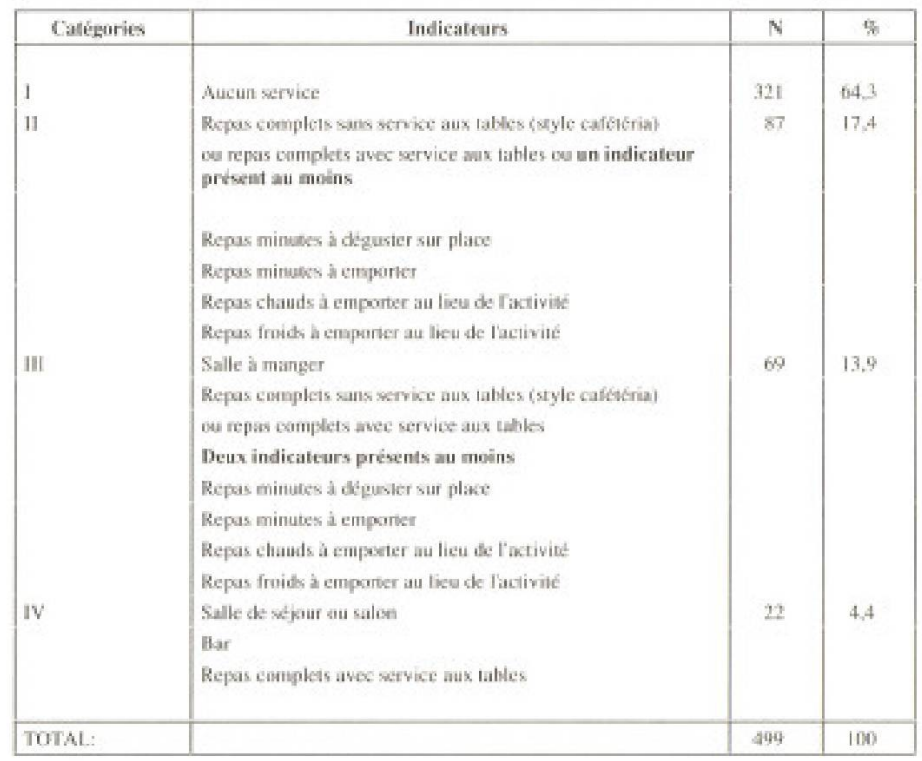

\section{TABLEAU 8}

Résultats comparés pour l'ensemble des pourvoiries du Québec incluses dans chaque classe selon la méthode de la moyenne et celle de la cote la plus basse

\begin{tabular}{|c|c|c|c|c|c|c|c|c|c|c|}
\hline \multirow[t]{2}{*}{ Cathoriks } & \multicolumn{2}{|c|}{$\begin{array}{l}\text { Camps } \\
\mathrm{V}=1106\end{array}$} & \multicolumn{2}{|c|}{$\begin{array}{l}\text { Chalets } \\
x=241 \mathrm{~s}\end{array}$} & \multicolumn{2}{|c|}{$\begin{array}{c}\text { Aaberges } \\
\text { Foredierres } \\
\mathrm{N}=78\end{array}$} & \multicolumn{2}{|c|}{$\begin{array}{l}\text { Motels } \\
\mathrm{N}=9\end{array}$} & \multicolumn{2}{|c|}{$\begin{array}{l}\text { Dortiniss } \\
\mathrm{N}=\mathrm{x}\end{array}$} \\
\hline & 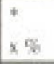 & $\mathrm{B} \%$ & & $B \%$ & & 4 & & B & $\begin{array}{l}* \\
x>5\end{array}$ & $8 \%$ \\
\hline 1 & 7,6 & 26 & $3, t$ & 14 & 0 & 0 & a & 0 & 6,7 & 6,7 \\
\hline II & 게:S & by 6 & 56,4 & 78.6 & 275 & 333 & Sil & 6.5 & 513 & PII \\
\hline IIII & Isil, 4 & 4 & 34,1 & 10,7 & 377 & 566 & 5n & 375 & 333 & 134 \\
\hline$I V$ & 1.2 & 0,4 & 5,4 & 0.7 & 348 & 110.1 & (1) & (1) & 4.7 & $a$ \\
\hline
\end{tabular}

* $\mathrm{x}=$ calcule aved la methode de la movene

$\mathrm{B}=$ calculé avee la méthode de la cote la plus basse

\section{TABLEAU 9}

Résultats comparés pour l'ensemble des pourvoiries du Québec incluses dans chaque classe selon la méthode de la moyenne et celle de la cote la plus basse

\begin{tabular}{|c|c|c|c|c|c|c|}
\hline \multirow[t]{2}{*}{ Classes } & \multicolumn{2}{|c|}{$\begin{array}{c}\text { Auberiges } \\
\text { foreatieres } \\
\mathrm{N}=78\end{array}$} & \multicolumn{2}{|c|}{$\begin{array}{l}\text { Pawilions } \\
\mathrm{N}=1.54\end{array}$} & \multicolumn{2}{|c|}{$\begin{array}{l}\text { Restaurants } \\
\qquad \mathrm{N}=12\end{array}$} \\
\hline & 3 & * & I & * & , & , \\
\hline & $x \%$ & B & $x$ of & $\mathrm{B}$ क & $x \%$ & 189 \\
\hline I & 0 & 4 & 0 & $0 T$ & 0 & 6 \\
\hline II & 192 & 350 & 433 & 530 & 9,0 & 63,6 \\
\hline III & 438 & 52,1 & 36.6 & 410 & 455 & 36,4 \\
\hline IV & 370 & 123 & 20,1 & 5,3 & 455 & 0 \\
\hline
\end{tabular}

${ }^{3} \mathrm{x}=$ calculé avec la múthode de la movenne

$\mathrm{B}=$ caleull avee la nothode de la cote la plus basse 